Background: Much research has been conducted to establish best practice in psychosocial interventions for mental illness. The challenge of ensuring that best practice is delivered to consumers has historically been left to program managers. This research contributes to the science of technology transfer: assessing the transferability, uptake and effectiveness of a comprehensive psychological intervention within routine mental health services.

TheIllnessManagementandRecoveryProgram(IMR) was developed as one of six evidence-based practices in the (US) National Evidence-based Practices Project. The paper reports on the implementation of the IMR in Newcastle, Australia, and outcomes from both Australian and US sites.

Methods: This study comprised a multicenter, international effectiveness study of a comprehensive, evidence-based psychosocial intervention for serious mental illness. The study centers included public mental health facilities in Newcastle and North Carolina. Rigorous fidelity and outcome measures were conducted at baseline, postintervention ( 9 months) and at follow-up (12 months).

Results: Retention in this comprehensive program was satisfactory $(76 \%)$. Statistically significant improvements were noted on a range of measures including the BSI, GAF, Coping Skills subscales, Knowledge of Illness, and the Recovery Assessment Scale.

Conclusions: The pilot data support the feasibility of implementing the IMR program in routine mental health settings. The findings suggest that participation in the program is associated with benefits in illness management, progress toward recovery and improved functioning. Individuals completing the IMR program reported significant improvements in hope, coping self-efficacy and distress-related to symptoms.

\section{Constructing longitudinal measures of maternal morbidity using a population health database: is use of the index birth record sufficient to identify obstetric risk factors for schizophrenia?}

\section{Croft, V Morgan, A Jablensky}

School of Psychiatry and Clinical Neurosciences, University of Western Australia, Perth, Australia

Background: Western Australia (WA) has extensive record-linked population health data collections. Midwives' records of WA births during 1980-1992 inclusive were linked and validated by the author to form sibships, which enable longitudinal family studies. Binary indicators of maternal morbidity were created using this database of prospectively collected birth data. A validated system, using case note reviews, for scoring obstetric complications as risk factors for psychiatric morbidity has been adapted for coding population data. This will be extended to 2001 births and include the mother's clinical history.

Methods: Women who have had only one birth during this period, who had multiple births or who had missing births in their sibship were excluded from analysis. Codes created reflect morbidity in the current pregnancy only, the one immediately prior, any prior pregnancy or, the current or any prior pregnancy.

Results: The database includes records of 472733 births to 249119 women with paternal data available for the majority of children. Of these, approximately $90000(36 \%)$ women were eligible for inclusion. Of the 67242 eligible women, who delivered during 1980-1992, severe underestimation of maternal conditions, affecting pregnancy, such as thyroid dysfunction, essential hypertension or epilepsy occurred when only index birth records were used.

Conclusions: Using data from the index birth only could underestimate the effects of maternal morbidity, as risk factors for outcomes such as schizophrenia, when compared with analysis of models that use longitudinal binary indicators of current and prior maternal illness.

\section{Altered tripartite synapse function: is this the problem in schizophrenia and bipolar disorder?}

\section{B Dean}

Rebecca L. Cooper Research Laboratories, Mental Health Research Institute, Melbourne, Australia

Schizophrenia and bipolar I disorder are serious psychiatric illnesses that affect approximately $4 \%$ of the Australian population. Both illnesses are thought to occur in individuals with a genetic predisposition after they encounter as yet unknown detrimental environmental factors. Postmortem central nervous system (CNS) studies have been undertaken to identify the combined impact of these two factors on both cellular and molecular cytoarchitecture. In particular, studies from our laboratories have shown changed levels of the glial-derived proteins apolipoprotein $\mathrm{E}$ and $\mathrm{D}$ in the cortex of subjects with schizophrenia and bipolar I disorder. In addition, we have shown changes in levels of $\mathrm{S} 100 \beta$, in the dorsolateral prefrontal cortex from subjects with bipolar II disorder. Significantly, it is known that levels of $\mathrm{S} 100 \beta$ are known to be potently regulated by serotonin through the serotonin $1 \mathrm{~A}$ receptor and receptors for apolipoprotein $\mathrm{E}$ are localized on neurons. Our data would therefore support the hypothesis that at least part of the pathology of schizophrenia and 